\title{
Wahl von DMV-Präsidiumsmitgliedern ab 2021
}

\author{
Vorstellung der Kandidatinnen und Kandidaten
}

Der Präsident der DMV ruft alle DMV-Mitglieder auf, sich an der Wahl von Präsidiumsmitgliedern für die Amtszeit ab dem 1.1.2021 zu beteiligen.

Bei der Wahl sind zunächst drei Präsidiumsplätze, die mit Funktionen versehen sind (Schatzmeister/in, Herausgeber/in der DMV-Mitteilungen - gleichzeitig Mitglieder im Vorstand der DMV - und Herausgeber/in des Jahresberichts), sowie zwei weitere Präsidiumsplätze zu besetzen. Für die Funktionsplätze gibt es jeweils eine Kandidatin bzw. einen Kandidaten. Für die weiteren zwei Präsidiumsplätze gibt es zwei Kandidatinnen und einen Kandidaten.

Die Kandidat/innen für die Funktionsplätze sowie die zwei weiteren Kandidat/innen mit den meisten Stimmen werden für eine Amtszeit von vier Jahren gewählt. Da Frau Ilka Agricola, derzeitiges Präsidiumsmitglied, vom Präsidium für die Amtszeit ab dem 1.1.2021 zur DMVPräsidentin gewählt wurde, wird ihre Position mit der dritten Kandidatin/dem dritten Kandidaten für die weiteren Präsidiumsplätze besetzt, und zwar für die verbleibende Amtszeit bis 31.12.2021, vgl. die DMV-Wahlordnung $\S 3(\mathrm{e})$. Im Jahr 2021 wird es erneut Präsidiumswahlen für die zum 1.1.2022 frei werdenden drei Präsidiumsplätze geben.

Die Kandidatinnen und Kandidaten stellen sich hier vor. Die Wahlzettel liegen diesem Heft bei.
Etienne Emmrich

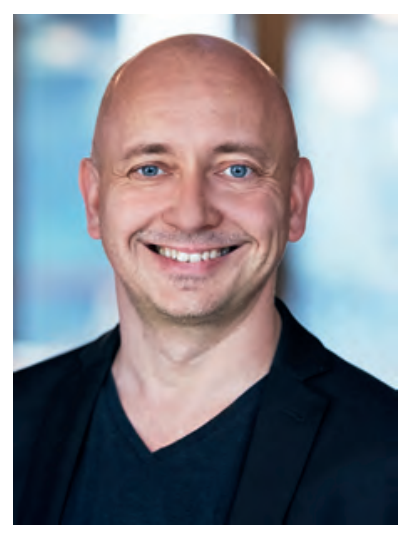

Geboren 1970 in Magdeburg. Studium in Magdeburg in der Fachrichtung Lehrer für Mathematik und Physik, Semester in Chemnitz und Amsterdam, Abschluss 1993 als Diplom-Mathematiker mit einer Arbeit zu analytischen und numerischen Aspekten hyperbolischer Erhaltungsgleichungen. Ab Oktober 1998 Wissenschaftlicher Mitarbeiter an

der TU Berlin und dort Promotion 2001 über Zeitdiskretisierungsverfahren für das inkompressible Navier-StokesProblem bei Rolf D. Grigorieff (Berlin) und Lutz Tobiska (Magdeburg). Danach Wissenschaftlicher Assistent bei Harry Yserentant (Berlin) und Habilitation 2007. Von Oktober 2009 bis Januar 2012 Professor für Mathematik, insbesondere Numerik, an der Universität Bielefeld. Seit Februar 2012 Professor für Mathematik, Arbeitsrichtung Differentialgleichungen, an der TU Berlin. Von 2013 bis 2017 Geschäftsführender Direktor des Instituts für Mathematik und seit 2017 Dekan der Fakultät II - Mathematik und Naturwissenschaften.

In meiner Forschung beschäftige ich mich mit nichtlinearen Evolutionsgleichungen, die die mathematische Beschreibung zeitabhängiger Prozesse erlauben. Im Vordergrund stehen dabei sowohl der Nachweis der Existenz verallgemeinerter Lösungen als auch die Analysis geeigne- ter Diskretisierungsverfahren mit Blick auf Anwendungen bei komplexen Fluiden und in der Elastodynamik. Ein weiterer Gegenstand meiner Forschung sind nichtlokale Effekte.

Neben der Forschung liegt mir die Ausbildung und Betreuung von Studierenden besonders am Herzen. Anderen Mathematik nahezubringen ist und bleibt für mich eine Aufgabe mit stets neuen Herausforderungen. Die Gestaltung von Übergängen (Schule-Hochschule, SchuleBeruf, Hochschule-Beruf) angesichts zunehmender Heterogenität und die Berücksichtigung der Bedürfnisse, der Schwächen und Stärken des einzelnen Studierenden im Massenbetrieb sehe ich auch weiterhin als eine der größten Herausforderung in der Lehre. Dies zeigt sich auch in der Arbeit der gemeinsamen Kommission von DMV, GDM und MNU zum Übergang Schule-Hochschule, in der ich seit einiger Zeit mitarbeite.

Mitglied der DMV bin ich seit 1994, Schatzmeister seit 2013. In meine Zeit als Schatzmeister fallen unter anderem eine Satzungsänderung, eine Beitragserhöhung und Änderung der Beitragskategorien, die Umstellung der Finanzbuchhaltung, die Neubesetzung der Geschäftsstelle und manch anderes. Derzeit arbeiten wir an der Umstellung der Mitgliedsverwaltung.

Um in der Bildungs- und Wissenschaftspolitik Einfluss zu nehmen und zu gestalten, braucht es eine starke Vertretung der Mathematik in Deutschland. Dafür halte ich es für erforderlich, mit anderen Fachgesellschaften eng zusammenzuarbeiten. Als Beauftragter der DMV für die Kontakte zur GAMM engagiere ich mich deshalb besonders für die Zusammenarbeit zwischen GAMM und DMV. 


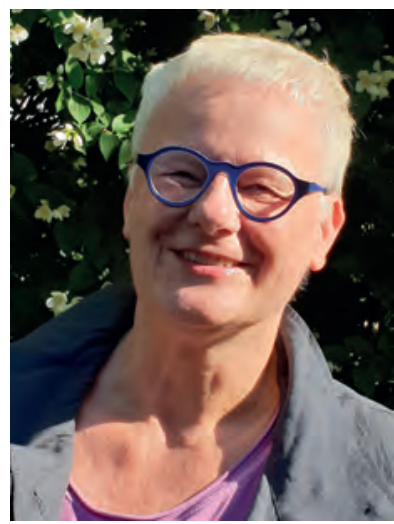

Geboren 1963 in Lüdenscheid. Studium der Mathematik mit Nebenfach Wirtschaftswissenschaften an der Universität Bielefeld, 1989 Diplom mit Schwerpunkt im Bereich Numerische Lineare Algebra, anschließend Studium an der State University of New York at Buffalo, USA, 1991 Master of Science mit Schwerpunkt Computer Science. Von 1991-200o an der Universität Bremen, dort 1994 Promotion und 1999 Habilitation, beides im Bereich der Numerischen Linearen Algebra. Von 2000-2002 Professur an der TU München, seit 2002 an der TU Braunschweig.

2004-2010 und seit 2017 Institutsleitung, 2007-2008 (Gründungs-)Dekanin der Carl-Friedrich-Gauß-Fakultät, 2008-2012 Vizepräsidentin für Lehre, Studium und Weiterbildung. Seit 2018 Mitglied im Hochschulrat der Bergischen Universität Wuppertal und im Akkreditierungsrat. Seit 2020 Mitglied des Scientific Council des Centre Européen de Recherche et de Formation Avancée en Calcul Scientifique (CERFACS).

In meiner Forschung beschäftige ich mich mit Fragestellungen aus dem Bereich der Numerischen Linearen und Multilinearen Algebra, insbesondere mit (strukturierten) linearen, polynomiellen und nichtlinearen Eigenwertproblemen und Matrixgleichungen, sowie Problemen aus dem Bereich der Modellreduktion. Zahlreiche der betrachteten Fragestellungen haben ihren Ursprung in industriellen Anwendungen, aber auch fundamentale Probleme in der Matrixtheorie liegen in meinem Interessensspektrum.

Anke Pohl

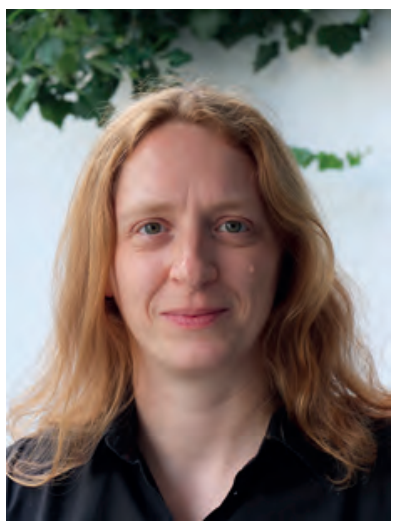

Geboren 1980 in Georgsmarienhütte, aufgewachsen in der Umgebung von Osnabrück. Studium der Mathematik mit den $\mathrm{Ne}$ benfächern Informatik und Wirtschaftswissenschaften an der TU Clausthal und der Universität Bologna, 2004 Abschluss mit Diplom. 2004-2009 wissenschaftliche Mitarbeiterin an der Universität Paderborn, 2009 Promotion im Bereich der dynamischen Systeme und Ergodentheorie. Danach wissenschaftliche Mitarbeiterin am Max-PlanckInstitut für Mathematik in Bonn, an der ETH Zürich und
Neben der Forschung liegt mir die Ausbildung und Betreuung von Studentinnen und Studenten besonders am Herzen. Anderen (insbesondere auch Studierenden aus den Ingenieur- und Naturwissenschaften) Mathematik nahezubringen ist und bleibt für mich eine Aufgabe mit stets neuen Herausforderungen. Neben der aktuell drängenden Frage, wie man dies digital gut umsetzen kann, beschäftigt mich schon seit langem die Frage nach der Gestaltung des Übergangs Schule-Hochschule und wie die Lehre im Massenbetrieb den immer weiter auseinanderdriftenden Bedürfnissen der Stärken und Schwächen der Studierenden gerecht werden kann.

Seit vielen Jahren engagiere ich mich in diversen mathematischen Fachorganisationen: u. a. bin ich seit 2008 Mitglied im Council der European Mathematical Society (EMS), 2017-2019 Präsidentin und 2020-2022 Vizepräsidentin der Gesellschaft für Angewandte Mathematik und Mechanik (GAMM), seit 2019 Schatzmeisterin des International Councils for Industrial and Applied Mathematics (ICIAM), seit 2020 Mitglied im Council der Society of Industrial and Applied Mathematics (SIAM). Daneben bin ich Mitorganisatorin zahlreicher wissenschaftlicher Tagungen, u. a. der DMV-GAMMJahrestagung 2016 in Braunschweig (gemeinsam mit Volker Bach).

Seit 2017 nehme ich als Gast (in meiner Funktion als (Vize-)Präsidentin der GAMM) an dem öffentlichen Teil der DMV-Präsidiumssitzung teil. Ich halte die Zusammenarbeit aller mathematischen Fachorganisationen in Deutschland für wichtig, um die jeweiligen Expertisen zu bündeln und gemeinsam für eine (noch) bessere Sichtbarkeit der Mathematik in der Öffentlichkeit und in der Politik einzutreten. Dafür würde ich mich gerne weiter einsetzen und kandidiere daher für einen der frei werdenden Präsidiumsplätze. der Universität Göttingen. 2016 Habilitation in Göttingen. 2016-2018 Professorin an der Universität Jena. Seit 2018 Professorin an der Universität Bremen.

Mein Forschungsinteresse gilt dem mathematischen Quantenchaos, der harmonischen Analysis und Spektraltheorie, den dynamischen Systemen und der Ergodentheorie sowie der Zahlentheorie. Insbesondere faszinieren mich die Wechselwirkungen zwischen diesen Gebieten, die es uns erlauben, viele tiefliegende Ergebnisse zu erzielen wie z. B. mathematische Inkarnationen des Korrespondenzprinzips zwischen klassischer Mechanik und Quantenmechanik. Ich bin (Mit-)Organisatorin zahlreicher wissenschaftlicher Tagungen und Sommerschulen zu diesen Themen.

Neben der Forschung ist mir auch die Lehre sehr wichtig. Hierbei ist es mir ein besonderes Anliegen, Studierenden nicht nur hochwertiges Fachwissen zu vermitteln, 
sondern ihnen auch zu ermöglichen, die Lebendigkeit und permanente Fortentwicklung der Mathematik zu erleben, einen entdeckenden Zugang zur Mathematik zu entwickeln und frühzeitig eigene Forschungserfahrungen zu sammeln. Zudem engagiere ich mich auf vielfältige Art in der Nachwuchsförderung, z. B. durch Seminare und Vortragsreihen für Schüler*innen und Studierende sowie als Vertrauensdozentin der Studienstiftung des deutschen Volkes.

Mathematik hat eine große Bedeutung für alle Aspekte unseres Lebens, manchmal auf eine verdeckte oder in-

Thomas Schick

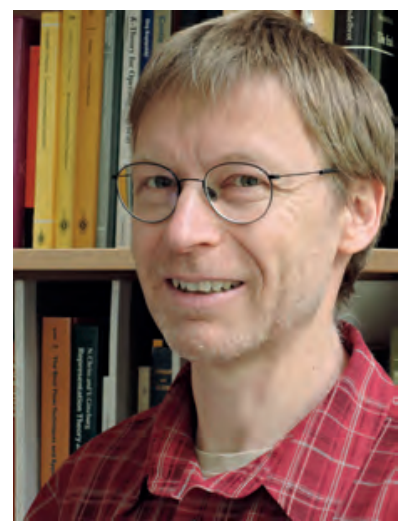

Geboren 1969 in Alzey, aufgewachsen in Rheinhessen. Studium der Mathematik und Physik in Mainz. 1994 Diplom und Promotion 1996 bei Wolfgang Lück in Mainz. 1996 bis 2001 wissenschaftlicher Assistent in Münster, dort 2000 Habilitation. Seit 2001 Professor für Geometrie in Göttingen. Forschungsaufenthalte und Gastprofessuren an der Penn State University

in State College, am MPI Bonn, ESI Wien, INI Cambridge, Hausdorff Institut Bonn, in Marseille, Clermont-Ferrand, Paris, Rom, Kopenhagen.

Mitorganisator zahlreicher wissenschaftlicher Tagungen, darunter auch Sektionen der DMV-Jahrestagung. Mitglied des wissenschaftlichen Beirats des Mathematischen Forschungsinstituts Oberwolfach seit 2014. Mitglied des Fachkollegiums Mathematik der DFG 2012-202O. Herausgeber der Mathematischen Annalen seit 2013.

Der Schwerpunkt meiner Forschung liegt im Bereich der geometrischen und algebraischen Topologie, insbe-

\section{Gudrun Thäter}

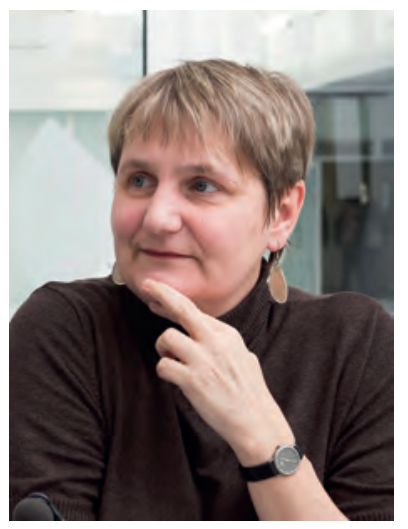

Gudrun Thäter, geboren 1964 in Weimar. Mathematikstudium TU Dresden, Spezialisierung auf Numerische Analysis unter Christian Großmann. Diplom 1987 mit Simulationen von Ionenimplantation für das Zentrum Mikroelektronik Dresden (ZMD). Geburten 1985 und 1987. 1987-1991 Entwicklungsingenieurin für Computer Aided Technolo- direkte Weise. Ebenso vielfältig gestalten sich daher die Aufgaben der DMV als Interessenvertretung der Mathematik und der Mathematiker*innen. Aus meiner Sicht sind dabei von besonderer Wichtigkeit: (1) die Stärkung des Bewusstseins der Gesellschaft für die Bedeutung und Schönheit der Mathematik, (2) die Interessenvertretung in der Bildungs- und Forschungspolitik und (3) die Vernetzung mathematisch Interessierter. Ich möchte die DMV gerne unterstützen, diese Aufgaben auch weiterhin erfolgreich zu bewältigen und kandidiere daher für einen der freiwerdenden Präsidiumsplätze.

sondere in ihren Wechselwirkungen mit der globalen Analysis und Operatoralgebren. Zudem interessiere ich mich für die Anwendungen in der theoretischen Physik. Über meine Forschung durfte ich als Sektionssprecher auf dem ICM 2014 in Seoul berichten. Seit 2016 bin ich ordentliches Mitglied der Göttinger Akademie der Wissenschaften.

Die Förderung des wissenschaftlichen Nachwuchses liegt mir sehr am Herzen, insbesondere in der Betreuung zahlreicher Diplom-, Bachelor- und Masterarbeiten und Promotionen. Aktuell bin ich Sprecher des Graduiertenkollegs 2491 „Fourieranalysis und Spektraltheorie“, vorher habe ich als Sprecher und stellvertretender Sprecher zweier anderer Graduiertenkollegs gewirkt. In der Lehre versuche ich, meine Begeisterung für die Mathematik auf die Studis zu übertragen, dabei engagiere ich mich gerne auch im Service in der Grundausbildung der Physik.

Bereits seit 1996 bin ich Mitglied der DMV und fungiere seit vielen Jahren als lokaler Ansprechpartner in Göttingen. Die DMV ist das wichtige Forum, in dem sich alle mathematisch Interessierten begegnen und austauschen können sollen. Sie hat die Expertise und das Mandat, die Anliegen der Mathematik in Gesellschaft und Politik zu vertreten. Hierfür bin bin gerne bereit, mich in Zukunft im Präsidium der DMV für unser Fach einzusetzen.

gy am ZMD. Hier erfuhr ich große Wertschätzung für die Beiträge der Mathematik und lernte jeden Tag von meinen Kollegen, die Physiker und E-Techniker waren - eine Erfahrung, die mich prägte.

1989 kündigten wir unserer Regierung und wurden anschließend vom Wandel aller Lebensbereiche erfaßt. Meine Familie verschlug es so 1991 nach Paderborn, wo wir 17 Jahre lang blieben und unsere Kinder groß werden sahen. Ab 1992 arbeitete ich an der Uni Paderborn zu Strömungstheorie. Dort schloß ich 1996 mit Herbert Sohr meine Promotion zur $L^{q}$-Helmholtzzerlegung und Regularität für den unendlichen Zylinder ab. 1997-2000 war ich am Mathematischen Seminar der Landwirtschaftlichen Fakultät der Uni Bonn in die Ausbildung von Geo- 
däsie Studierenden eingebunden, bevor ich 2000-2004 ans Institut für Angewandte Mathematik der Uni Hannover wechselte. Dort war ich ab 2001 Stipendiatin des „Dorothea-von-Erxleben-Programmes" und habilitierte $2004 \mathrm{zu}$ „Natural Convection, Dissipation and Power-law Rheology: Mathematical Models and Results". 2004-2008 sammelte ich vielfältige Erfahrungen als Hochschuldozentin am Fachbereich Mathematik der Uni Dortmund. Mit Aussicht auf eine Dauerstelle wechselte ich 2008 als Akademische Mitarbeiterin mit besonderen Aufgaben an das Institut für Mathematik der Uni Heidelberg und zog mit meinem Mann nach Heidelberg. Anfang 2009 ergriff ich die Möglichkeit, nach Karlsruhe an eine TU zu wechseln und fand dort etwas später auch ein neues Zuhause für uns. Seitdem bin ich im Institut für angewandte und numerische Mathematik an der Schnittstelle von Modellbildung und Anwendungen der Mathematik tätig. Die Strömungstheorie bietet mir die Möglichkeit, mit sehr unterschiedlichen Kolleginnen und Kollegen in den Ingenieurfakultäten zusammen zu arbeiten und durch 3d-Simulationen gibt es sogar Fragen, die wir im Zentrum für Medienkultur in Karlsruhe vorstellen. Beson- ders greifbar ist die Arbeit als Brückenbauerin für mich einerseits als Mitglied der Lattice Boltzmann Research Group zwischen Verfahrenstechnik und Mathematik und andererseits im Graduiertenkolleg SiMET, das den Lebenszyklus von Akkumulatoren auf allen Skalen untersucht und simuliert. Gerade bauen wir für interdisziplinäre Themen mit Mathematik am KIT einen besonderen Rahmen: das KIT-Zentrum MathSEE (Mathematics in Sciences, Engineering, and Economics), wo sich Mathematik und forschende Anwender auf Augenhöhe begegnen und der wissenschaftliche Nachwuchs in den je unterschiedlichen Kulturen der Fächer hochwillkommen und geschätzt ist.

Um von der Vielfalt der Arbeit und der Menschen in der Mathematik zu erzählen, schufen wir 2013 den Podcast Modellansatz, in dem mein Kollege Sebastian Ritterbusch und ich Gespräche mit Kolleginnen und Kollegen führen. Als Nebenprodukt setzt sich in mittlerweile über 230 Folgen auch ein facettenreiches Bild der Rolle von Mathematik für uns und die Gesellschaft zusammen. Als Herausgeberin der Mitteilungen werde ich dieses Mosaik der Mathematik für uns als Mitglieder der DMV in den Mittelpunkt stellen.

Alexander Zimmermann

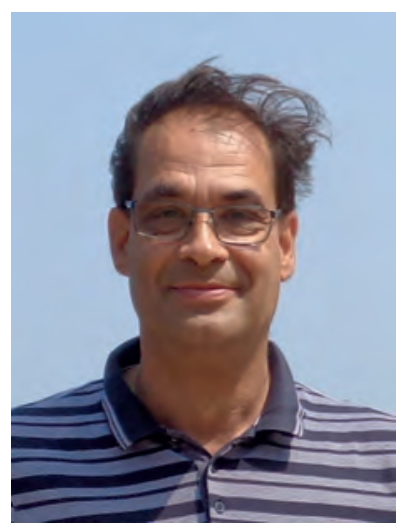

Geboren 1964 und aufgewachsen bin ich in Stuttgart. Ich bin verheiratet und habe zwei erwachsenen Kinder. Mein Studium der Mathematik (Diplom) mit Nebenfach Physik in Stuttgart schloss ich 1990 ab und wurde dort anschließend 1992 mit einem Thema zur ganzzahligen Darstellungstheorie endlicher Gruppen promoviert. Von 1990 bis 1994 war ich dort wissenschaftlicher

Mitarbeiter und erhielt ab November 1994 bis September 1996 ein DFG Postdoktoranden-Stipendium. Von April 1995 bis September 1996 war ich damit zu einem Forschungsaufenthalt an der École Normale Supérieure in Paris sowie an der Université Paris 7 Denis Didérot in der Arbeitsgruppe bei Michel Broué und Raphaël Rouquier, wo ich in engem Kontakt mit Bernhard Keller stand. Im September 1996 wurde ich an die Université de Picardie in Amiens/Frankreich zum Maître de Conférences berufen. Die „Habilitation à Diriger des Recherches“ fand im Dezember 1998 statt. Im September 2004 erhielt ich die Berufung zum Professeur des Universités in Amiens. Es folgten dann 2012 und 2019 Beförderungen in die nun höchst mögliche Klasse Professeur Classe Exceptionnelle jeweils auf Entscheidung des nationalen Komitees CNU.

Schwerpunkt meiner wissenschaftlichen Arbeit ist die Darstellungstheorie von Gruppen und assoziativen
Algebren. Dabei geht es darum, abstrakte Gruppen beziehungsweise Algebren mittels Realisierungen als konkrete Matrizengruppen beziehungsweise Matrizenalgebren $\mathrm{zu}$ verstehen. Bei fester Gruppe beziehungsweise Algebra untersuche ich nicht einzelne solcher Realisierungen, sondern eher die Gesamtheit all dieser Realisierungen in Form besonders strukturierter Kategorien. Die abstrakte Methode, die mich dabei besonders interessiert, entstand aus der algebraischen Geometrie und algebraischen Topologie, und entsprechend ergeben sich zahlreiche Beziehungen $z u$ diesen und anderen Gebieten bis hin zu Aspekten der mathematischen Physik.

Wir leben in einer spannenden Zeit. Unsere Wissenschaft entwickelt sich in rasender Geschwindigkeit, alte teilweise seit Jahrhunderten offene Probleme werden gelöst, und zahlreiche der neueren technologischen und gesellschaftlichen Entwicklungen entstehen aus sehr unterschiedlichen Disziplinen der Mathematik. Einige schöpfen ihre Methoden aus Bereichen der Mathematik, die bis vor kurzem noch jeder technischen Anwendung fern lagen. So facettenreich wie die Mathematik sollte auch das Themenspektrum des Jahresberichts sein.

Herr Guido Schneider hat den Jahresbericht aus meiner Sicht sehr erfolgreich geleitet. Ich werde versuchen, den Jahresbericht in dieser Richtung weiterzuentwickeln, und gemeinsam mit einem möglichst breiten Mitherausgebergremium fachlich variantenreich und verständlich über mathematische Entwicklungen zu berichten. Zudem finden historische Artikel und die Buchbesprechungen ihren wichtigen Platz. 